\title{
Effect of surface functionalities on the methylene blue adsorption capacity of feather fiber-derived multi-doped carbon
}

\author{
Poonam Benjwal ${ }^{1}$, Raghunandan Sharma ${ }^{2}$ and Kamal K. Kar ${ }^{1,2^{*}}$ \\ ${ }^{1}$ Advanced Nanoengineering Materials laboratory, Materials Science Programme, \\ ${ }^{2}$ Advanced Nanoengineering Materials laboratory, Department of Mechanical Engineering, \\ Indian Institute of Technology Kanpur, Kanpur-208016, India \\ *Email:kamalkk@iitk.ac.in
}

The discharge of dyes from the textiles industries into the aquatic environment has triggered many problems to the public health due to their toxic nature and carcinogenicity. Thus, various efforts have been made in past for removal of dyes, where the adsorption method is recognized as one of the most promising and cost-effective method. For adsorption process, the activated carbon is the most popular choice due to its effectiveness and versatility and thereby it has been widely used for the treatment of wastewater

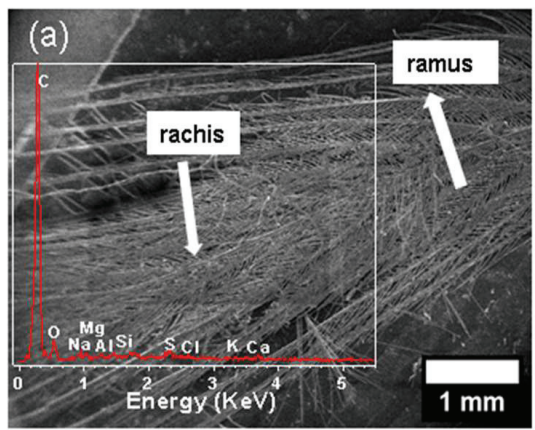

Figure 1: The SEM image of ramus and rachis parts of $\mathrm{CFF}$

However, the limitation with commercially available activated carbon is its cost as it is generally derived from the wood or coal. Chicken featherfibers (CFFs), composed of mostly keratin, a form of natural protein are produced in large amounts from the poultry processing (Figure 1). Therefore, in the quest of production of value added products from CFF, significant efforts are being made. On the heat treatment of CFFs in inert atmosphere, microporous carbon fibers containing high fractions of nitrogen can be produced.

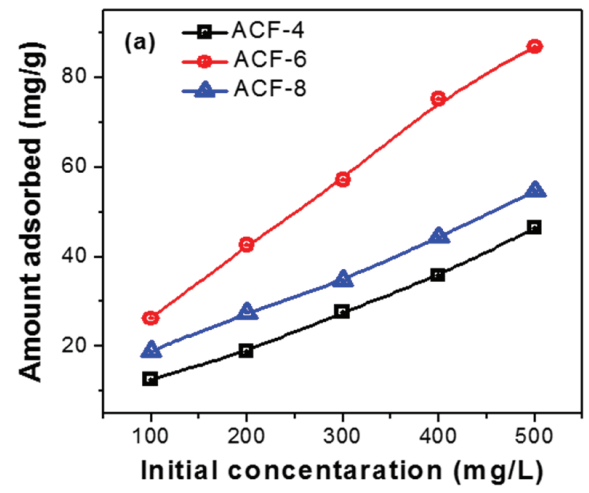

Figure 2: Effect of concentration of $\mathrm{MB}$ on adsorption over ACF

Here, an attempt is made to evaluate the potential of CFF based activated carbon (ACF) for the removal of methylene blue (MB) dye from the aqueous solution. ACF was prepared by the pyrolysis of CFF at temperatures of 400 , 600 and $800{ }^{\circ} \mathrm{C}$. The as-synthesized ACF consists of surface bound nitrogen groups, which play a major role for the adsorption of MB dye. Thus, an objective of the present study is also to explore the role of surface adsorbed nitrogen groups in the removal of pollutants. The results are explained in terms of the adsorption capacity (Figure 2). Further, the effects of solution $\mathrm{pH}$, dye concentration, contact time on the MB adsorption are investigated. 